Nusgens, B., Lambert Ch., Lapière Ch.M.

\title{
Signaling through rho gtpases in microgravity (rho signaling) on iss (soyuz tma-1) belgian soyuz mission "odissea"
}

Rho GTPases, RhoA, Racl and Cdc42, are molecular switches in the intracellular signaling pathways, that relay the information collected by receptors to soluble mediators and insoluble extracellular matrix environment. The objective of this experiment was to investigate the impact of microgravity on cellular processes depending on Rho GTPases activity, i.e.cytoskeleton and focal adhesions organization, GTPases translocation to the membrane, nuclear translocation of signaling molecules and gene expression. WI26 fibroblasts were stably transfected by the constitutively active form of each of the Rho GTPase. Selected clone of the engineered cells and the wild-type cells were used during the belgian ODISSEA Soyuz mission to investigate the alterations of the mechanical and phenotypic expression of the cells induced by microgravity and their rescue by the engineered Rho GTPases. A failure in the time schedule, a disconnection of the experiment containers before the automatic activation of the fixation procedure, was responsible for the loss of the biological samples.

Authors

Nusgens, B., Lambert Ch., Lapière Ch.M.

University of Liège, Laboratory of Connective Tissues Biology,

BELGIUM

+3243662456; 1ctb@ulg.ac.be

\section{Introduction}

Adhesion, spreading, migration and several other cell processes including gene expression rely on the function of integrins, the signaling pathway initiated in the focal adhesions and the dynamics of the cytoskeleton. The small $\mathrm{G}$ proteins of the Rho family (Rho GTPases) are key operators in the integration of signals arising from integrins and membrane receptors for diffusible ligands. RhoA, Rac1 and $\mathrm{Cdc} 42$ operate as binary molecular switches flipping from an inactive GDP-bound to an active GTP-bound form under the activity of exchange factors. The resulting conformational changes induce the activation of a panel of downstream effectors responsible for the control of several signaling pathways including the organization of the cytoskeleton, the focal adhesions and the associated integrins as well as cell proliferation, survival and gene expression. Data obtained during the STS-95 mission showing that microgravity affects cytoskeleton architecture and gene expression (increased MMP1 and IL-6) support a potential disturbance of the function of Rho GTPases in a weightlessness environment.

The main objective of this experiment was to analyze by immunomorphological and biochemical procedures distinct cellular processes depending on Rho GTPases activity, i.e cytoskeleton and focal adhesions organization, GTPases translocation to the membrane, translocation of activated signaling molecules and gene expression. Their functional alteration in microgravity was investigated in human fibroblasts and their involvement supported by a correction of the weightlessness effects in similar fibroblasts stably transfected by constitutively activated form of each of the three GTPases, RhoA, Rac1 and $\operatorname{Cdc} 42$.

Our experiments used human WI26 fibroblasts, a cell line behaving similarly to strains of skin fibroblasts. This cell line proved suitable for stable transfection of plasmids containing the consitutively active form generated by glutamine-to leucine mutation (QL) of each of the three GTPases. The most relevant clones were selected by functional pull-down assay $[1,2]$. 
Ground experiments using the selected clones of the QL transfected cells and/or suppression of the Rho GTPases by small interfering RNA (si-RNA) clearly demonstrated their expected biological activity. The fibrillar actin organization pattern after staining with FITC-conjugated phalloidin was characterized by the presence of filopodia and few thin actin filaments in cells expressing Cdc42 QL, lamellipodia in cells expressing Rac1 QL while RhoA QL cells exhibited robust actin stress fibers. The latter pattern was accompanied by a significantly increased detergent-insoluble vinculin indicating the formation of large focal adhesions [2]. In agreement with the pattern of actin fibers, time-lapse video microscopy showed a significant directed migration with a speed of $0.27 \mu \mathrm{m} / \mathrm{min}$ for the Rac1 QL while the RhoA QL cells did not significantly move [3]. These data were strengthened by observing opposite behaviour in cells silenced for RhoA, Rac1 and Cdc 42 by specific siRNAs. Furthermore, it was demonstrated that $\mathrm{Cdc} 42$ exerts a negative regulation on the expression of MM1P, a matrix metalloproteinase involved in connective tissues degradation [4].

The experiment of the "Odissea" Belgian Soyuz mission (Rho signaling) was devised to investigate the effect of microgravity on the cellular functions depending on the cytoskeleton and their correction by the constitutively active RhoGTPases (QL).

The WI26 wild type (non transfected) and the RhoA QL, Rac 1 QL and Cdc42 QL were plated at a subconfluent density on Permanox slides coated with polymeric type I collagen. Each type of cells was used in triplicate. Post flight analysis was planned to use one quarter of each slide for immunodetection of actin stress fibers and vinculin in the focal adhesions to check for reproducibility in the aspect of the cytoskeleton. The other

A
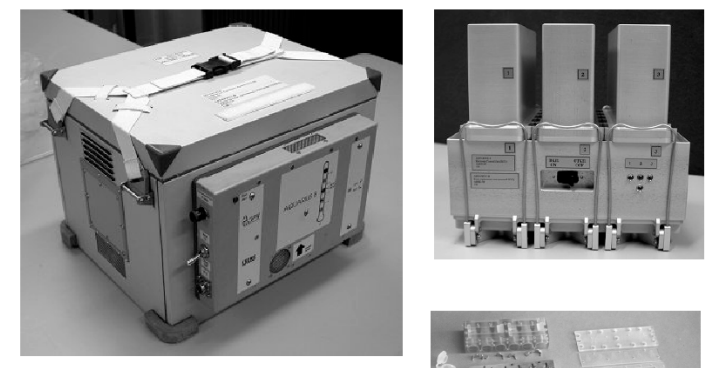

C

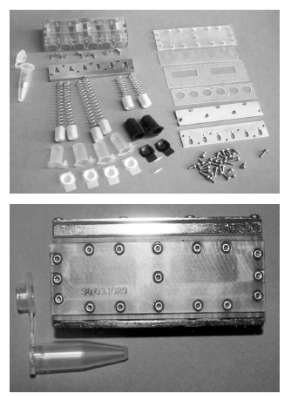

quarters were planned to be used for detection of other markers, tubulin and vimentin, phosphotyrosine containing proteins, localisation of the Rho GTPases, interference microscopy to detect signs of degradation of the pericellular fibrous matrix. MMP1, IL-1 and IL-6 contained in the spent medium at the end of the experiment were to be measured by ELISA.

The Rho signaling experiment was carried to the ISS in November 2002 and executed on the ISS, within the Aquarius B Transport/Ascent Incubator (CTA-B, Fig. 1A). The CTA-B is a thermally controlled and isolated container for storage of biological experiments, with a Peltier element actively controlling the temperature of the inner chamber $\left(22\right.$ and $\left.37^{\circ} \mathrm{C}\right)$. Besides the Rho signaling experiments, two other experiments, Vitamin D and Ramiros were housed in the CTA-B. Each experiment was located in a B-container (Fig 1B and C), a vacuum tight housing made in aluminium, providing 2 levels of containment for the plunger box units (PBU, Fig. 1D) and their contents.

The 6 Rho signaling PBUs were fully automated and contained 2 culture compartments and six cylindrical storage compartments each ( 2 of them filled with fixative). The transfer of the liquid was performed by means of a plunger, driven by a pre-loaded spring, which is released by a heater-wire system (mini pyro-cutter) according to a pre-programmed experiment activation time. After docking of the Soyuz to the ISS at L +2.5 days, the astronaut moved the CTA-B from Soyuz to the ISS. The experiment was activated automatically via the electronic control unit (ECU) and required no crew intervention until the completion of the experiment. This is indicated by the flashing of a green light. Two-days after ECU switch-on, the CTA-B was opened, the Rho signaling experiment B-container status inspected and then removed. The container was attached to the outside of the CTA-B, to allow it to cool from $37^{\circ} \mathrm{C}$ to ambient and transferred to the Aquarius B Transport/Return Incubator (CTR-B), a passive version of Aquarius B Incubator for return back to Earth.

Upon inspection of the PBUs after landing and transfer to our research unit, it turned out that plungers $\mathrm{A}$ and $\mathrm{D}$ of each unit had been correctly activated to make the exchange of culture medium 6 hours after reaching $37^{\circ} \mathrm{C}$ in the incubator. Plungers $\mathrm{B}, \mathrm{C}, \mathrm{E}$ and $\mathrm{F}$ activating the washing and the fixation of the samples had not been activated, a failure responsible for the loss of biological samples. Post-flight debriefing revealed that the Bcontainer was disconnected and removed from the Aquarius 5 minutes before termination of the experiment just before the activation of the plungers $\mathrm{B}, \mathrm{C}$ and $\mathrm{E}, \mathrm{F}$.

To conclude, additional security should be applied in the future to prevent avoidable failures.

Fig. 1: A: Aquarius B Transport/Ascent Incubator (CTA-B); B: Bcontainer; $C$ : three $B$ containers housed in the CTA-B; D: plunger box unit (PBU) before and after assembly 
B. Nusgens et al: Signaling through rho gtpases in microgravity (rho signaling) on iss (soyuz tma-1) belgian soyuz mission "odissea"

\section{References}

(1) Deroanne C., Vouret-Craviari V. Wang B. and Pouyssegur J. EphrinA1 inactivates integrin-mediated vascular smooth muscle cell spreading via the Rac/PAK pathway. J Cell Science, 116: 1367-76, 2003.

(2) Servotte S., Zhang Z., Lambert Ch.L., Ho T.T.G, Chometon G., Eckes B., Krieg Th., Lapière Ch.M., Nusgens B.V. and Aumailley M. Establishment of stable human fibroblast cell lines constitutively expressing active RhoGTPases. Protoplasma 229 (2-4), 215-220, 2006.

(3) Zhang Z., Lambert Ch.L., Servotte S., Chometon G., Eckes B., Krieg Th., Lapiere Ch.M., Nusgens B.V., Aumailley M. Effects of constitutively active GTPases on fibroblast behavior. Cell Mol Life Sci, 63: 82-91, 2006.

(4) Deroanne C, Hamelryckx D, Ho GTT, Lambert C, Catroux P, Lapière ChM and Nusgens, B. Cdc42 downregulates MMP-1 expression by inhibiting the ERK1/2 pathway. J Cell Sci, 118: 1173-1183, 2005. 